Saudi Journal of Medical and Pharmaceutical Sciences

Abbreviated Key Title: Saudi J Med Pharm Sci ISSN 2413-4929 (Print) |ISSN 2413-4910 (Online) Scholars Middle East Publishers, Dubai, United Arab Emirates Journal homepage: https://saudijournals.com/sjmps

\title{
An Insight into the Corona Virus Disease 2019 (COVID 19)
}

\author{
Pratiush Saxena*, Dr. Atul K. Gangwar
}

S.R. Institute of Pharmacy, Bhuta, Bareilly, U.P. 243001, India

DOI: $10.36348 /$ sjmps.2020.v06i04.007

| Received: 15.04.2020 | Accepted: 22.04.2020 | Published: 25.04.2020

*Corresponding author: Pratiush Saxena

\section{Abstract}

The corona virus disease 2019 is a type of pneumonia which is found to be fatal. The novel corona virus named as SARS CoV 2 which emerged from Whuan, China in December 2020 is now spread in more than 200 countries and has lead to more than 40,000 fatalities worldwide. The COVID 19 hence stood up as the most dangerous disease with highest fatalities in the world. The taxanomy of this novel virus is not very similar to other corona virus (MERS CoV and SARS $\mathrm{CoV}$ ) which have also affected humans. Thus it has created a situation where we don't have any such antiviral drug which can be effective against this virus during its incubation in human body. The spread of this novel virus is so rapid that it took only couple of months to spread to almost whole world. The symptoms are as common as cold, cough; sore throat and lastly pneumonia which are very normal and similar to influenza infection so were not treated seriously during initiation of spread. As there is no prevention and cure for this novel virus and no first line treatment for COVID 19 the only way to combat this disease is to stop the spread which can only be done by social distancing which is also being advised by WHO.

Keywords: COVID 19, taxanomy, Whuan, social distancing.

Copyright @ 2020: This is an open-access article distributed under the terms of the Creative Commons Attribution license which permits unrestricted use, distribution, and reproduction in any medium for non-commercial use (NonCommercial, or CC-BY-NC) provided the original author and source are credited.

\section{INTRODUCTION}

The disease referred to as corona virus disease 2019 (abbreviated as COVID 19) has been declared as pandemic by WHO, as because of its high frequency of transmission. Corona virus is named so because of the crown like spikes on their surface. Corona virus is generally classified into four types' alpha, beta, gamma and delta [1]. Human corona virus were first identified in mid of 1960, there are seven types of corona virus strains that can infect humans out of which four are the common human corona virus and three are those from which the infection occurs to human but are new in emergence.

\section{Common human coronaviruses}

1. 229E (alpha coronavirus)

2. NL63 (alpha coronavirus)

3. OC43 (beta coronavirus)

4. HKU1 (beta coronavirus)

\section{Other human coronaviruses}

5. MERS-CoV (the beta coronavirus that causes Middle East Respiratory Syndrome, or MERS)

6. SARS-CoV (the beta coronavirus that causes severe acute respiratory syndrome, or SARS)
7. SARS-CoV-2 (the novel coronavirus that causes coronavirus disease 2019, or COVID-19)

First four types 229E, NL63, OC43, and HKU1 are the most common coronavirus that cause infection to humans. Sometimes coronaviruses that infect animals can evolve and make people sick and become a new human coronavirus. Three recent examples of this are 2019-nCoV, SARS-CoV, and MERS-CoV. Most commonly all types of coronavirus infect the respiratory tract and cause infection.

\section{Emergence and identification}

In December 2019, the emergence of novel corona virus has been conformed after the investigation of Chinese health authorities in Wuhan Hubei Province, China. Cluster of atypical pneumonia cases occurring primarily in individuals who had visited a seafood and wet market have been reported. Patients reported fever and cough, and most developed chest discomfort and/or respiratory distress, with a diagnosis of pneumonia being made by chest radiographs and/or computed tomographic (CT) scan [2]. After testing for common causes of respiratory infection yielded negative results, unbiased sequencing of bronchoalveolar lavage (BAL) fluid identified a variant beta-coronavirus with nearly $85 \%$ sequence homology to that of a bat severe acute 
respiratory syndrome (SARS)-like coronavirus $(\mathrm{CoV})[2]$. The virus was subsequently isolated in eukaryotic cell culture, and further characterization showed it to be distinct from SARS-CoV and Middle East respiratory syndrome (MERS)-CoV, with sequence homology of approximately $79 \%$ and about $50 \%$, respectively [3]. The variant $\mathrm{CoV}$, which has been named SARS-CoV-2 by the International Committee on Taxonomy of Viruses [4], represents the seventh CoV to cause disease in humans, and the third $\mathrm{CoV}$ since 2003 to cross over from animals to humans and be associated with severe respiratory illness (1). The World Health Organization (WHO) has named the illness caused by SARS-CoV-2 coronavirus disease2019 (COVID-19).

\section{Taxonomy}

Coronaviruses (CoVs) belong to the subfamily Orthocoronavirinae in the family Coronaviridae and the order Nidovirales. CoVs have an enveloped, crown-like viral particle from which they were named after. The $\mathrm{CoV}$ genome is a positive-sense, single-strand RNA (+ssRNA), 27-32 kb in size, which is the second largest of all RNA virus genomes. Typically, two thirds of the genomic RNA encodes for two large overlapping polyproteins, ORF1a and ORF1b, that are processed into the viral polymerase (RdRp) and other nonstructural proteins involved in RNA synthesis or host response modulation. The other third of the genome encodes for four structural proteins (spike (S), envelope (E), membrane (M), and nucleocapsid (N) and other accessory proteins. While the ORF1a/ORF1b and the four structural proteins are relatively consistent, the length of the $\mathrm{CoV}$ genome is largely dependent on the number and size of accessory proteins [5].

Compared with other RNA viruses, the expanded genome size of CoVs is believed to be associated with increased replication fidelity, after acquiring genes encoding RNA-processing enzymes ${ }^{6}$. Genome expansion further facilitates the acquisition of genes encoding accessory proteins that are beneficial for CoVs to adapt to a specific host [7]. As a result, genome changes caused by recombination, gene interchange, and gene insertion or deletion are common among CoVs. The CoV subfamily is expanding rapidly, due to the application of next generation sequencing which has increased the detection and identification of new $\mathrm{CoV}$ species. As a result, $\mathrm{CoV}$ taxonomy is constantly changing. According to the latest International Committee of Taxonomy of Viruses (ICTV) classification, there are four genera $(\alpha-, \beta-, \delta$, and $\gamma-)$ consisting of thirty-eight unique species in the subfamily [7]. The number of species will continue to increase, as there are still many unclassified CoVs [8, 9].

CoVs cause disease in a variety of domestic and wild animals as well as in humans, where $\alpha$ - and $\beta$ CoVs mainly infect mammals and $\gamma$ - and $\delta$-CoVs mainly infect birds (Table 1). Two highly pathogenic $\beta$ CoVs, SARS-CoV, and MERS-CoV have caused pandemics in humans since 2002[10, 11]. Originating in China and then spreading to other parts of the world, SARS-CoV infected around 8000 individuals with an overall mortality of $10 \%$ during the 2002-2003 pandemic [11]. Since its emergence in 2012 in the Middle East, MERS-CoV spread to 27 countries, resulting in 2249 laboratory-confirmed cases of infection with an average mortality of $35.5 \%$ (until September 2018) [9]. Besides these two viruses, $\alpha$ CoVs 229E and NL63 and $\beta$-CoVs OC43 and HKU1 can also cause respiratory diseases in humans [12]. Moreover, CoVs cause pandemic disease in domestic and wild animals (Table 1). SADS-CoV was recently identified as the etiological agent responsible for a large-scale outbreak of fatal disease in pigs in China that caused the death of more than 20,000 piglets. Porcine epidemic diarrhea virus (PEDV) and transmissible gastroenteritis virus (TGEV) that belong to $\alpha-\mathrm{CoV}$ and porcine $\delta-\mathrm{CoV}$ (PDCoV) are also important emerging and re-emerging viruses in pigs that pose significant economic threat to the swine industry [13]. In addition, avian infectious bronchitis virus (IBV, $\gamma-\mathrm{CoV}$ ) causes a highly contagious disease that affects poultry production worldwide [14]. Coronaviruses have also been associated with catarrhal gastroenteritis in mink (MCoV) and whale deaths (BWCoV-SW1)[15, 16].

\section{Spread}

A previously unknown coronavirus, named SARS-CoV-2, was discovered in December 2019 in Wuhan, Hubei province of China and was sequenced and isolated by January 2020. SARSCoV- 2 is associated with an ongoing outbreak of atypical pneumonia (Covid-2019) that has affected over $8,58,371$ people and killed more than 40,000 of those affected in more than 200 countries as of March 31, 2020. On January 30, 2020, the World Health Organization declared the SARS-CoV-2 epidemic a public health emergency of international concern.

MERS-CoV was suggested to originate from bats, but the reservoir host fueling spillover to humans is unequivocally dromedary camels. Both SARS-CoV and SARS-CoV-2 are closely relatedand originated in bats, who most likely serve as reservoir hostfor these two viruses. Whereas palm civets and racoon dogs have been recognized as intermediate hosts for zoonotic transmission of SARS-CoV between bats and humans, the SARS-CoV-2 intermediate host remains unknown. The recurrent spillovers of coronaviruses in humans along with detection of numerous coronaviruses in bats, including many SARS-related coronaviruses (SARSr$\mathrm{CoVs}$ ), suggest that future zoonotic transmission events may continue. In addition to the highly pathogenic zoonotic pathogens SARS-CoV, MERS-CoV, and SARS-CoV-2, all belonging to the b-coronavirus genus, 
four low-pathogenicity coronaviruses are endemic in humans: HCoV-OC43, HCoVHKU1, HCoV-NL63, and
HCoV-229E. To date, no therapeutics or vaccines are approved against any human-infecting coronaviruses.
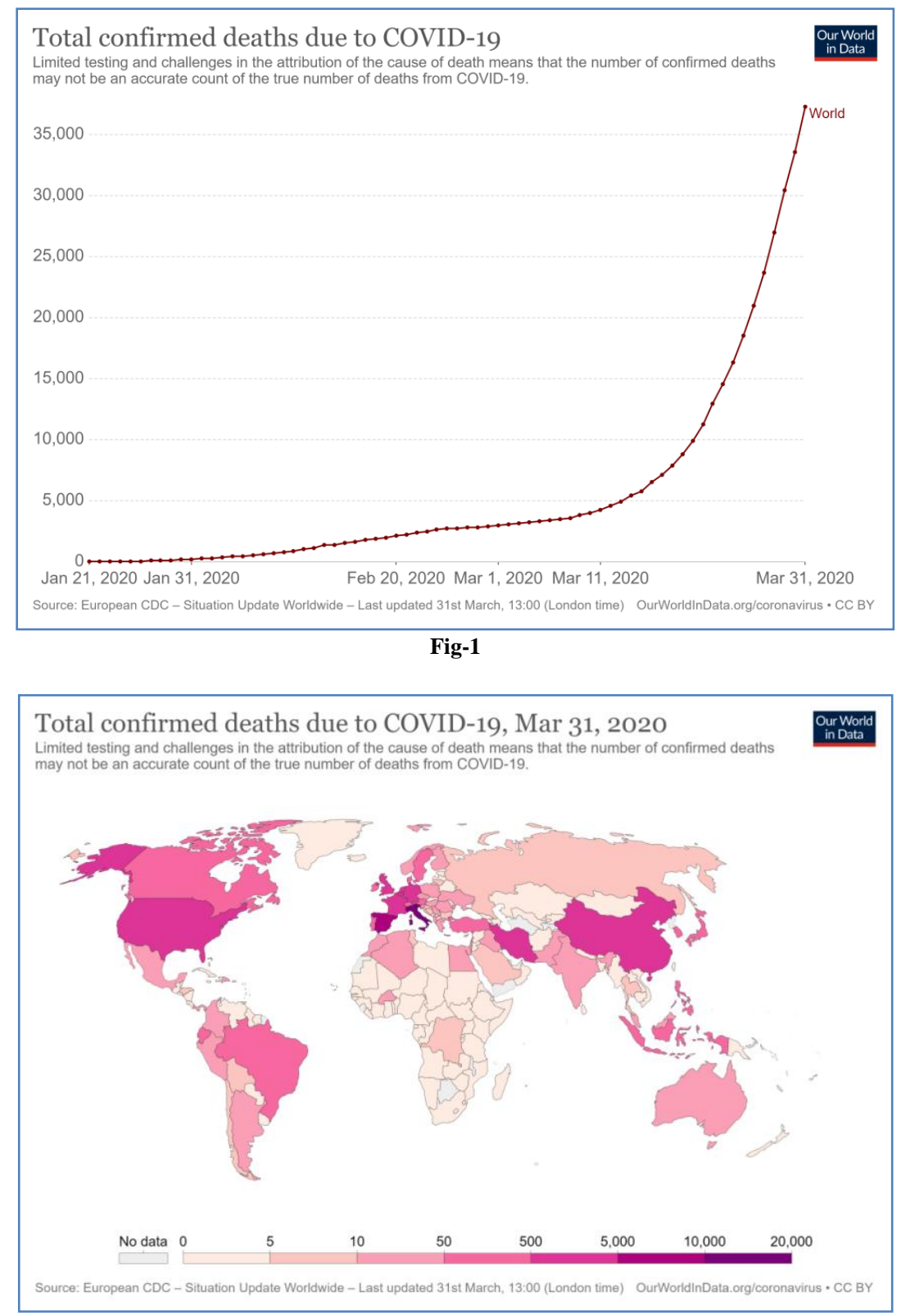

Fig-2

\section{CONCLUSION}

The outburst of the COVID 19 stood up as the most fatal pandemic since the virus is novel there is no medicine that can cure the disease. The spread of the virus is so fast by human to human contact that it leaves take any preventive action to produce vaccine. The criticality with SARS CoV 2 is that the symptoms that were in initial stage were very similar to cold, cough and pneumonia that the healthcare professionals were unable to identify the causative agent is a novel corona virus. The only way to fight with COVID 19 is to maintain the social distancing, which is only the way to break up the chain of the growth and spread of SARS $\mathrm{CoV} 2$ i.e. the novel corona virus. WHO has advised all effected countries to take up measures to maintain social distancing by various means? The conditions may be very unfavorable and fatal for those countries which will not maintain social distancing by any means which will lead to spread this virus and may lead to fatality.

\section{REFERENCES}

1. Centers for Disease Control and Prevention. Human corona virus types https://www.cdc.gov/coronavirus/types.html

2. Zhu, N., Zhang, D., Wang, W., Li, X., Yang, B., Song, J., \& Niu, P. (2020). A novel coronavirus 
from patients with pneumonia in China, 2019. New England Journal of Medicine.

3. Lu, R., Zhao, X., Li, J., Niu, P., Yang, B., Wu, H., \& Bi, Y. (2020). Genomic characterisation and epidemiology of 2019 novel coronavirus: implications for virus origins and receptor binding. The Lancet, 395(10224), 565-574.

4. Gorbalenya, A. E. (2020). Severe acute respiratory syndrome-related coronavirus-The species and its viruses, a statement of the Coronavirus Study Group. BioRxiv.

5. Coronavirinae in Viral Zone. (2019). Available online: https://viralzone.expasy.org/785 (accessed on 28 January 2019).

6. Subissi, L., Posthuma, C. C., Collet, A., Zevenhoven-Dobbe, J. C., Gorbalenya, A. E., Decroly, E., ... \& Imbert, I. (2014). One severe acute respiratory syndrome coronavirus protein complex integrates processive RNA polymerase and exonuclease activities. Proceedings of the National Academy of Sciences, 111(37), E3900E3909.

7. Forni, D., Cagliani, R., Clerici, M., \& Sironi, M. (2017). Molecular evolution of human coronavirus genomes. Trends in microbiology, 25(1), 35-48.

8. ICTV Virus Taxonomy. (2018). Release. 2018. Available online: https://talk.ictvonline.org/taxonomy/ (accessed on 28 January 2019).

9. Ge, X. Y., Wang, N., Zhang, W., Hu, B., Li, B., Zhang, Y. Z., \& Wang, B. (2016). Coexistence of multiple coronaviruses in several bat colonies in an abandoned mineshaft. Virologica Sinica, 31(1), 3140.

10. Wu, Z., Yang, L., Ren, X., He, G., Zhang, J., Yang, J., \& Du, J. (2016). Deciphering the bat virome catalog to better understand the ecological diversity of bat viruses and the bat origin of emerging infectious diseases. The ISME journal, 10(3), 609620.

11. Drosten, C., Günther, S., Preiser, W., Van Der Werf, S., Brodt, H. R., Becker, S., \& Berger, A. (2003). Identification of a novel coronavirus in patients with severe acute respiratory syndrome. New England journal of medicine, 348(20), 1967-1976.

12. Graham, R. L., Donaldson, E. F., \& Baric, R. S. (2013). A decade after SARS: strategies for controlling emerging coronaviruses. Nature Reviews Microbiology, 11(12), 836-848.

13. Zhang, Q., \& Yoo, D. (2016). Immune evasion of porcine enteric coronaviruses and viral modulation of antiviral innate signaling. Virus research, 226, 128-141.

14. Zhao, Y., Zhang, H., Zhao, J., Zhong, Q., Jin, J. H., \& Zhang, G. Z. (2016). Evolution of infectious bronchitis virus in China over the past two decades. Journal of General Virology, 97(7), 15661574.

15. Mihindukulasuriya, K. A., Wu, G., Leger, J. S., Nordhausen, R. W., \& Wang, D. (2008). Identification of a novel coronavirus from a beluga whale by using a panviral microarray. Journal of virology, 82(10), 5084-5088.

16. Vlasova, A. N., Halpin, R., Wang, S., Ghedin, E., Spiro, D. J., \& Saif, L. J. (2011). Molecular characterization of a new species in the genus Alphacoronavirus associated with mink epizootic catarrhal gastroenteritis. The Journal of general virology, 92(Pt 6), 1369. 